\title{
RECIEN
}

Revista Cientifica de Enfermeria

\section{VARIABILIDAD DE LA ESTANCIA MEDIA EN PACIENTES HOSPITALIZADOS EN UN HOSPITAL DE TRAUMATOLOGÍA DURANTE EL AÑO 2010}

\section{VARIABILITY OF AVERAGE OF STAY IN PATIENTS HOSPITALIZED IN THE TRAUMATOLOGY HOSPITAL DURING THE YEAR 2010}

\author{
VÍCTOR MANUEL GONZÁLEZ-CHORDA ${ }^{a, b}$ \\ PABLO SALAS-MEDINA ${ }^{a, b}$ \\ DESIREE MENA TUDELA ${ }^{a, b}$
}

(a) Departamento de Enfermería.

(b) Universitat Jaume I. Castellón de la Plana. España.

E-mail contacto: vchorda@uji.es 


\section{RESUMEN}

\section{Titulo:}

Variabilidad de la estancia media en pacientes hospitalizados en un hospital de Traumatologia durante el año 2010.

\section{Objetivos:}

El objetivo principal del estudio es conocer la estancia media hospitalaria y su variabilidad en relación con el motivo de ingreso, en una unidad de hospitalización de un hospital monográfico de Traumatología. (Unión de Mutuas. Castellón)

\section{Metodología:}

Estudio transversal, descriptivo, basado en datos administrativos, cuya población estuvo formada por todos los individuos que tuvieron algún episodio de hospitalización durante el año 2010, siendo este el criterio de inclusión, por orden cronológico de aparición.

\section{Resultados:}

Se identificaron un total de 736 episodios asistenciales y 133 códigos CIE-9CM. La edad media fue $44(+/-10)$ años. El 83,25\% de los pacientes fueron varones. El código 836.0 (Desgarro de cartílago o menisco interno de la rodilla-actual) fue el diagnóstico más habitual $(12,25 \%)$ y la artroscopia la técnica quirúrgica mayoritaria. "Fractura de pelvis" (CIE-9CM 808) resultó el código diagnóstico que presentó mayor estancia media con 25 (+/-9.84) estancias y mayor variabilidad de la misma, seguido de los diagnósticos "Fractura de diáfisis de tibia-cerrada", "Fractura de diáfisis de tibia/peronéabierta", "Contusión de múltiples sitios, ncoc" y "Fractura de tibia y peroné".

\section{Conclusiones:}

Se observa poca variabilidad en la estancia media excepto en los procesos diagnosticados como fractura de pelvis y en los relacionados con fracturas de los miembros inferiores.

\section{Palabras clave:}

Variabilidad, Estancia media, GRD, Grupos de pacientes relacionados por el diagnóstico, enfermería. 


\section{ABSTRACT}

Title:

Variability of average of stay in patients hospitalized in the Traumatology hospital during the year 2010.

\section{Objectives:}

The main objective of the study is to identify the average hospital stay and the variability of in connection with the reason for admission in a hospital unit.

\section{Methodology:}

Cross-sectional, descriptive, whose population consisted of all individuals who had an episode of hospitalization during 2010, this being the criterion for inclusion, in chronological order of appearance.

\section{Results:}

736 episodes of care and 133 ICD-9CM were identified. The average age was $44(+/-10)$ years. $83.25 \%$ of the patients were male. The code 836.0 (torn cartilage or meniscus of the knee-current) was the most common diagnosis (12.25\%) and arthroscopic surgical technique majority. Fractured pelvis (ICD-9CM 808) was the diagnostic code had a higher average stay of 25 $(+/$-9.84) placements.

\section{Conclusions:}

There is little variability in average of stay except processes diagnosed as a fracture of pelvis and those related to fractures of the lower limbs.

\section{Key words:}

Variability, length of stay DRG, Diagnosis related-groups, nursing. 


\section{INTRODUCCIÓN}

Los estudios descriptivos relacionados con la estancia media hospitalaria como el que se presenta, son un punto de partida para investigaciones relacionadas con la calidad asistencial que, aportan información de interés en la detección de problemas directos relacionados con la calidad y en ocasiones orientan sobre causas de variabilidad en determinados procedimientos 0 tecnologías sanitarias.

La duración de cada episodio de hospitalización (estancia), es el indicador de resultado que se considera actualmente que, mejor internaliza los gastos directos de la asistencia sanitaria, explicando la posible variabilidad ${ }^{1,2}$ de los mismos. El indicador estancia media ha sido utilizado en ocasiones para, explicar la variabilidad en la práctica clínica ${ }^{3-5}$ ya que desde hace algunos años, la literatura científica revela la existencia de factores no relacionados con los pacientes, que modifican la estancia hospitalaria6-7.

El uso de herramientas relacionadas con la calidad asistencial como las guías clínicas (GC) que recopilan la evidencia sobre una determinada patología o proceso clínico, ordenando la información en una secuencia lógica para los profesionales de la salud o la utilización de vías clínicas (VC) para seguimiento de procesos de evolución predictible han demostrado la disminución de la variabilidad en la práctica clínica, la estancia media hospitalaria y los costes por proceso $8-10$.

El servicio de hospitalización del Instituto de Traumatología de Unión de Mutuas (ITUM) de Castellón de la Plana, comprometido con la mejora de la calidad, tiene planteados objetivos orientados hacia sus clientes y potencia los estudios de calidad asistencial.

Este estudio forma parte del inicio de un proceso de mejora .Consiste en realizar un análisis descriptivo que incluya características de los clientes, patologías más frecuentes y su estancia media, con objeto de observar la variabilidad intrahospitalaria, valorar sus posibles causas y la necesidad de implantar herramientas de mejora como las ya mencionadas. Como objetivos 
secundarios se han propuesto estudiar las características sociodemográficas de los pacientes, conocer los diagnósticos y procedimientos más frecuentes, y detectar la existencia de posibles errores en los datos contenidos en el CMBD.

\section{MÉTODO}

Se trata de un estudio transversal, descriptivo, basado en datos administrativos de registro, cuya población estuvo formada por todos los pacientes que tuvieron algún episodio de hospitalización durante el año 2010 en el ITUM, siendo este el criterio de inclusión.

El ITUM se ubica en la ciudad de Castellón de la Plana (España). Su dependencia funcional y patrimonial corresponde a Unión de Mutuas. Posee un total de 28 camas de hospitalización instaladas, siendo su finalidad asistencial la traumatología y/o rehabilitación.

Fueron excluidos del estudio los episodios con una duración inferior a 1 estancia (24 horas), por considerarse episodios ambulatorios, fallecimientos antes de cumplir el primer día de ingreso o traslados urgentes a otros centros. También se excluyeron aquellos episodios sin la información necesaria para llevar a cabo la investigación, y los episodios, que aun siendo atendidos por el mismo personal, fueron ingresados en la Unidad de Cirugía Ambulatoria (UCA).

Se tuvo acceso a los datos de toda la población. Se llevó a cabo un muestreo consecutivo de casos, por orden cronológico, de todos los episodios de hospitalización del año 2010. La fuente de datos principal fue la historia clínica digitalizada de Unión de Mutuas, a través del software CHAMAN.

Se solicitó a Unión de Mutuas acceso a los datos del CMBD y de las historias clínicas, aportando una copia del protocolo de investigación y un compromiso ético de los investigadores. Se obtuvo informe favorable, poniendo la entidad como requisito la previa disociación de los datos clínicos y personales de los pacientes atendidos, tal y como prevé la Ley Orgánica 15/1999 de Protección de Datos de Carácter Personal, y por la no existencia de una autorización expresa de cada cliente para poder acceder a los datos contenidos en sus respectivas historias clínicas informatizadas para fines 
investigadores. La entidad facilitó una base de datos con la mayoría de las variables solicitadas para el estudio, sin ningún dato de carácter personal. No se autorizó el acceso a las historias clínicas digitales. En la tabla 1 se recogen las variables que finalmente fueron objeto de estudio.

El análisis de los datos se realizó a partir de la base de datos facilitada por Unión de Mutuas. Se realizó un análisis descriptivo de todas las variables cualitativas y cuantitativas, para el conjunto de episodio y en función de los códigos diagnósticos según la clasificación CIE-9MC. Se consideraron para las variables cuantitativas las medias y desviaciones típicas, además de las medianas y los cuartiles. El análisis de las variables cualitativas se realizó a través del cálculo y análisis de proporciones, con sus intervalos. La desviación típica de las estancias medias de los diferentes códigos diagnósticos CIE-9MC, fue el estadístico utilizado para estudiar la variabilidad de la estancia media de los procesos asistenciales. Se realizó un estudio por grupos quinquenales de edad. Para el análisis de los datos se utilizó la aplicación Rcomander del software R. El nivel de significación estadística fue de $p<0.05$

La población a estudio (ingresos en la unidad de hospitalización durante el año 2010) se preveía escasa para llevar a cabo un estudio en profundidad de la variabilidad de la estancia media por proceso, siendo esta una de las principales limitaciones metodológicas de esta investigación. Por otra parte, no se realizó el cálculo de la estancia media ajustada por factores relacionados con el paciente (edad, sexo, comorbilidad, etc) el proceso (complicaciones) o la institución (médico).

No se identificó ningún tipo de conflicto entre los principios bioéticos fundamentales, por parte de los investigadores, en el desarrollo de esta investigación de carácter descriptivo. No se identificaron conflictos de intereses por parte de los autores. 


\section{RESULTADOS}

\section{Episodios.}

Se identificaron un total de 736 episodios asistenciales durante 2010. Un episodio fue excluido por no contener la información de las variables Estancia, Estancia preoperatoria y Estancia postoperatoria. 205 episodios asistenciales fueron excluidos por pertenecer a otra institución (27,85\%), y 130 por tener estancia inferior a 24 horas $(17,66 \%)$. No se excluyó ningún episodio por no contener la información relacionada con la variable "diagnóstico principal", quedando para el análisis un total de 400 episodios asistenciales de hospitalización, con estancia superior a 24 horas, pertenecientes al año 2010, $(54,49 \%)$.

Los meses de mayor actividad fueron Febrero $(12,75 \%)$ y Marzo $(12 \%)$ con 51 y 48 episodios asistenciales, respectivamente; Agosto y Septiembre fueron los meses con menos ingresos durante el año 2010. Se observa un ligero descenso de la actividad en el segundo semestre del año 2010.

Tipo de pacientes y motivos de ingreso.

De acuerdo a la nomenclatura interna, al agrupar los datos de pacientes, el $86,25 \%$ de los episodios fueron ingresados como programados con intervención $(n=345)$, el $7,5 \%$ fueron urgencias con intervención $(n=30)$ y el $6,25 \%$ restante se repartía entre los ingresos programados sin intervención $(n=20)$ y urgencias sin intervención que requirieron ingreso $(n=5)$.

De los 400 episodios asistenciales, el $4,5 \%$ fueron reingresos $(n=18)$ y el $9.94 \%$ se clasificaron como reintervención $(n=27)$. En el $88,75 \%$ de los episodios el origen correspondía a Unión de Mutuas $(n=355)$ y 45 episodios provenían de otro origen. No se identificó ningún episodio de hospitalización, con más de una estancia, cuyo origen correspondiese a Corporación Mutua (grupo de mutuas al que pertenece Unión de Mutuas), durante el año 2010. No se identificó ningún reingreso con carácter urgente. 
Tipo de lesión motivo de ingreso.

Se identificaron 263 episodios de hospitalización por accidente laboral (65\%), 74 ingresos por enfermedad común, 18 por accidentes in itínere-tráfico y 45 clasificados como otros. En $97,75 \%$ episodios los pacientes fueron dados de alta por curación $(n=391)$. Siete episodios se clasificaron como alta domicilio.

Cumplimentación de información.

En 81 episodios asistenciales clasificados como programados con intervención, el $23 \%$ de los episodios correspondientes a esta categoría, faltaba alguna información de registro.

La técnica anestésica se registró en el $70 \%$ de los episodios clasificados como programados sin intervención $(n=14)$ y en un $25 \%$ de esta categoría se recuperó información sobre la técnica quirúrgica empleada $(n=5)$

El procedimiento principal se identificó en 78 de los 400 episodios asistenciales pertenecientes a hospitalización (19\%). Hay que considerar que, hasta el segundo semestre del año 2010 no se completó la transferencia de la información a la base de datos informatizada, quedando los registros anteriores en formato papel.

\section{Codificación de diagnósticos CIE-9MC:}

Se identificaron un total de 133 códigos diagnósticos diferentes. El código 836.0 (Desgarro de cartílago o menisco interno de la rodilla-actual) fue el diagnóstico más habitual presentándose en el $12.25 \%$ de los casos $(n=49)$, seguido del código 717 (Trastorno interno de rodilla) en el 6,50\% de episodios $(n=26)$ y los códigos 719.46 (Dolor articular-pierna) y 844 (Esguince y torcedura de rodilla y pierna) con 16 episodios respectivamente (4\%).

En la tabla 2 pueden observarse los 25 códigos CIE-9MC con mayor número de casos, relacionados con la estancia media, además de las estancias medias preoperatorias y postoperatorias. 
Técnicas y su frecuencia.

La artroscopia de rodilla se empleó en el 7,25\% de los episodios asistenciales $(n=29)$, siendo el procedimiento más utilizado, seguido de la reducción abierta de fractura con fijación interna $(n=10)$ en el $2,5 \%$ y la artroscopia $(n=8)$ en el $2 \%$ de los episodios asistenciales. Todos los procedimientos principales recuperados fueron quirúrgicos a excepción de los relacionados con la comunicación de pacientes (entrevista, evaluación, consulta y examen), identificados en un paciente.

\section{Estancia media y variabilidad.}

La estancia media del centro fue de $1,87(+/-3,37)$ días, con 1 día como estancia mínima y 36 días de estancia máxima. La estancia media preoperatoria fue de $0.34(+/-1,93)$ días, con un máximo de 35 días en un solo caso y 0 estancias como mínimo. La estancia media postoperatoria fue de 1,52 $(+/-2,34)$ días, con una estancia máxima postoperatoria de 23 días y mínima de 1 día.

La estancia media más elevada corresponde a Fractura de pelvis (CIE9 CM 808) con 25 (+/-9.84) estancias, seguido del CIE-9CM 923.10 (Contusión del antebrazo) con una estancia media de $24(+/-0)$ días.

La Fractura de pelvis presentó una mayor variabilidad en la estancia media del total de procesos asistenciales estudiado, con un mínimo de 17 estancias y un máximo de 36 estancias. Su estancia media preoperatoria fue de 13,66 (+/-18.47) estancias, con un mínimo de 3 y un máximo de 35 estancias. Este diagnóstico fue el que presentó mayor variabilidad en este sentido y en la estancia media postoperatoria con $11,33(+/-9,29)$ estancias de media.

En la Tabla 3 pueden consultarse los cinco códigos CIE-9CM que presentaron mayor variabilidad en la estancia media total, la estancia media preoperatoria y la estancia media postoperatoria. 


\section{Edad.}

La edad media de los pacientes fue de 44 años (ds 10), oscilando entre los 18 y los 80 años. El $83,25 \%$ de los pacientes fueron varones $(n=333)$ y el resto mujeres $(n=67)$.

En el estudio por grupos quinquenales de edad, el grupo más representativo fue el de $36-40$ años $(n=66)$, con un $16.5 \%$ del total de episodios, seguido de 41-45 años con 62 episodios, $15.5 \%$ del total. Los grupos menos representativos fueron menores de 20 años y mayores de 65, con 3 episodios de hospitalización durante el año 2010 cada uno. Los pacientes entre 41 y 45 años fueron los que tuvieron más ingresos programados con intervención ( $n=60)$, siendo entre 36 y 40 años los que tuvieron más ingresos programados sin intervención. Respecto a los ingresos urgentes sin intervención, fue el grupo 51-55 años el que tuvo más ingresos de esta categoría $(n=9)$, seguido del grupo de edad 31-35 años $(n=7)$. Los grupos de edad 26-30 y 41-45 años, fueron los que tuvieron más episodios urgentes sin intervención.

Los pacientes del grupo 31-35 años fueron los que tuvieron más reingresos $(n=6)$ y entre 46 y 50 años, el grupo con más reintervenciones $(n=6)$. Respecto a la estancia media, el grupo de edad entre 36 y 40 años fue el que presentó mayor estancia media, mayor estancia postoperatoria y mayor variabilidad con $3.1(+/-5.68)$ y $2.53(+/-4.65)$ estancias, respectivamente.

El código CIE-9CM (Desgarro de cartílago o menisco interno de la rodillaactual) fue el más representativo en los grupos 31-35 y 41-60 años. En el grupo 36-40 años el diagnóstico más representativo fue Trastorno interno de rodilla (CIE-9CM 717). 


\section{DISCUSIÓN}

Se identificaron los diagnósticos con mayor número de casos, mayor estancia media total, mayor estancia media preoperatoria y mayor estancia media postoperatoria, y los que tenían mayor variabilidad (ver Tabla 2 y 3 ).

Igualmente se identificaron 133 códigos diagnósticos en 400 episodios asistenciales. El CIE-9CM 808 (Fractura de pelvis) fue el diagnóstico con mayor estancia media y mayor variabilidad en ésta, seguido de otros diagnósticos relacionados con patologías de los miembros inferiores, aunque la variabilidad en éstos fue menor.

La accesibilidad a la información y el método de codificación aportan información de calidad en términos de fiabilidad e identificación de problemas lo que garantiza posibilidad de realizar investigación y mejora de calidad. Sin herramientas básicas, resulta complejo estudiar el uso inadecuado de la hospitalización o la variabilidad asistencial como elementos de calidad 11,12,13.

Existe literatura reciente que relaciona variabilidad en la práctica clínica y prolongación de la estancia hospitalaria ${ }^{14}$. Igualmente el uso inapropiado de la hospitalización se considera un elemento de no calidad.

En el estudio, no parece existir una variabilidad significativa en las estancias medias (preoperatoria y postoperatoria) del conjunto de procesos estudiados diferentes a estudios realizados en otros Centros de características similares ${ }^{15}$.

La Norma Estatal de GRD-2010 del conjunto de hospitales españoles reporta una estancia media depurada inferior a 8 estancias para los GRD quirúrgicos relacionados con la fractura de pelvis (GRD 334, GRD 335) ${ }^{16}$. En este estudio se reporta una estancia media de 25 (+/-9.84) estancias, triplicando el dato reportado por la Norma Estatal. Llama la atención la elevada estancia preoperatoria de estos procesos asistenciales, con una media de $13,66(+/-18,47)$ estancias. Las posibles causas de la prolongada estancia media de la fractura de pelvis y su variabilidad, se estudiaran posteriormente ya que son multicausales ${ }^{15}$.

Se han identificado errores de codificación de los datos contenidos en el CMBD. En 2005 se llevó a cabo un estudio en el Servicio Vasco de Salud (SVS) 
que analizó los datos del CMBD de los años 2003 y 2004, identificando un porcentaje de error en la codificación de algunos procedimientos quirúrgicos del $8 \%$, disminuyendo este error en un $50 \%$ tras aplicar durante un año una aplicación informática creada "ad hoc"17. Posteriormente, en 2008, se realizó otra investigación ${ }^{18}$ en el SVS que estudió la calidad de las variables administrativas del CMBD, encontrando un porcentaje de error que oscilaba entre el 0,7\% para la variable "sexo" y el $24,9 \%$ para la variable "médico responsable". Existen estudios en ambos sentidos; en algunos casos se observa un elevado reporte de errores y en otros una pequeña cantidad pero justificada ${ }^{19}$.

Respecto a las limitaciones del estudio, el método utilizado para la obtención y análisis los de datos (base de datos facilitada por la entidad disociando datos personales datos clínicos) por una parte, agilizó el desarrollo de la investigación ya que la recogida de datos se llevó a cabo de forma informatizada; como contrapartida conllevó la pérdida de información en alguna variable (Procedimiento principal) y la exclusión de alguna de las variables propuestas inicialmente (Alergias, Comorbilidades, Complicaciones).

El tamaño de la muestra ha resultado escaso para llevar a cabo un estudio en profundidad de la variabilidad de la estancia media ya que se han identificado 400 episodios para 133 códigos diagnósticos, por lo que existen diagnósticos con sólo dos registros, restando valor al cálculo de las desviaciones típicas. La realización de un estudio con series temporales más largas permitiría el uso de otros estadísticos de variabilidad como el coeficiente de variación. El uso de la estancia media no ajustada limita las conclusiones del estudio al no considerar otras variables que pueden influir en los resultados, por lo que sería conveniente llevar a cabo un estudio añadiendo factores como comorbilidades o complicaciones.

Antes de comenzar a estudiar las causas de la variabilidad en la estancia media, parece recomendable realizar estudios en profundidad multicentricos, ya que podría ayudar a identificar los puntos débiles de la codificación, permitiendo una mejora en la calidad de los datos contenidos en el CMDB.

Este estudio realiza una primera aproximación tanto sobre la variabilidad de la estancia media, como del case-mix atendido en el centro hospitalario. 


\section{CONCLUSIONES}

En el Instituto Traumatológico de Unión de Mutuas (ITUM) de Castellón, se atienden principalmente procesos asistenciales quirúrgicos, relacionados con la traumatología y en especial con los miembros inferiores, siendo la artroscopia de rodilla la técnica predominante. Los usuarios del servicio de hospitalización son principalmente hombres, con una edad media de 44 años y una estancia media aproximada para el conjunto de procesos de 48 horas, que desciende a 24 horas en los procesos asistenciales más habituales, con un ingreso hospitalario igual o superior a 1 día.

Se observa, en general, poca variabilidad en la estancia media hospitalaria, así como en la estancia media preoperatoria y postoperatoria, excepto en los episodios diagnosticados como fractura de pelvis y aquellos relacionados con fracturas de los miembros inferiores que son, en conjunto, los que presentan mayor la estancia media (total, preoperatoria y postoperatoria).

Unión de Mutuas cuenta con guías clínicas médicas de diferentes patologías y regiones anatómicas (hombro, rodilla, tobillo, lumbalgia, cervicalgia y dorsalgia).

Un estudio más profundo sobre las causas de variabilidad en los procesos de fractura de pelvis y fracturas de tibia y peroné, ayudaría en la toma de decisiones relacionadas con la mejora de la calidad asistencial.

\section{BIBLIOGRAFÍA}

1. Controlling health care expenditure: some recent experiences with reform. Report ECFIN/157/04-EN. Bruselas: European Union, Directorate General for Economic and Financial Affairs. 2004.

2. Cots, F; Castells, X; García, A; Sáez M. Relación de los costes directos de la hospitalización con la duración de la estancia. Gac Sanit. 1997; 11:285-95.

3. Chen, E; Naylor, CD. Variation in length of stay for acute myocardial infartion on Canada. Med Care.1994;32:420-34.

4. Lutjens, LR. Determinants of hospital lengths of stay. J Nurs 
Adm.1993;23:14-8.

5. Aróstegui, I; Quintana, JM; Arcelay, A. Uso del PMC en el estudio de la varaibilidad de la estancia media hospitalaria en tres procesos quirúrgicos. Gac Sanit.1998;12:169-75.

6. Burns, LR; Wholey, DR. The effect of patient, hospital and physician characteristics on lenght of stay and mortality. Med Care. 1991;29:251-71.

7. Jiménez Paneque, RE; Guitérrez Rojas, AR; Fariñas Seijas, H; Suárez García, $\mathrm{N}$; Fuentes Valdés, E. Variaciones del tiempo de estancia postoperatoria según las características de los pacientes en un servicio de cirugía general. Gac Sanit. $1994 ; 8: 180-8$.

8. Soria-Aledo, $\mathrm{V}$ et al. Evaluación de la vía clínica de la colecistectomía laparoscópica. Cir Esp. 2005; 77(2):86-90. [Consultado el 01.10.2010] Disponible en http://scielo.isciii.es/pdf/gs/v18n3/original5.pdf.

9. Esteve, M; Serra-Prat, M; Zaldívar, C; Verdaguer, A; Berenguer, J. Evaluación del impacto de una trayectoria clínica sobre el ictus isquémico. Gac Sanit $2004 ; 18(3): 197-204$

10. Pérez Blanco, V; García Caballero, J; Ureña Vilardell, V; Martinez, L; Díez Sebastián, J; Reoyo, A; Rodriguez, E. Evaluación de una vía clínica para la apendicitis infantil tras dos años de implantación. Rev. Calidad Asistencial. 2007;22(3):113-7.

11. Antón, P; Peiró, S; Martínez Pillado, M; Aranaz Andrés, JM. Efectividad de las intervenciones de revisión de la utilización inadecuada de la hospitalización. Una revisión sistemática. Rev Calidad Asistencial. 2008;23(5): 236-44.

12. Sanders, D;Coulter, A; Mcpherson, K. Variation in hospital admission rates, a rewiev of the literature. Londres: King Edward's Hospital Fund. 1989.

13. Moreno García, F; Sánchez Ramiro, MA; Alejandre Lázaro, G; Rubio Pulio, O; Fernández Agüero, L; Villarin Castro, A; Viseu Pinheiro Lopes do Regoa, MG. ¿También somos variables ante la evidencia? Rev Clin Med Fam [Înternet]. 2010. [Consultado el 21.11.2011] Diponible en http://www.revclinmedfam.com/articulo.php?pagina $=0 \& a r t=107$ 
14. González Chordá, VM; Maciá Soler, ML. Grupos de pacientes Relacionados por el Diagnóstico (GRD) en los hospitales generales españoles: variabilidad en la estancia media y el coste medio por proceso. E Global. 2011; 10(24). [Consultado el 17.11.2011] Disponible en http://revistas.um.es/eglobal/article/view/134471

15. Moreno Millán, E; García Torrecillas, JM; lea Pereira, MC. Variaciones de la estancia media preoperatoria en España según grupos de eda, sexo y modo de acceso hospitalario (urgente o programado). Rev Calidad Asistencial. 2008; 23(5): 222-229.

16. Ministerio de Sanidad, Servicios Sociales e Igualdad. Norma Estatal de GRD (AP-GRD V25) para el conjunto de hospitales del Servicio Nacional de Salud. Año 2010. [Consultado el 14.04.2.10]. Disponible en http://www.msps.es/estadEstudios/estadisticas/cmbd.htm

17. Olive, F; Gomez, F; Schott, AM; Remontet, L; Bossard, N; Mitton, N; Polazzi, S; Colonna, M; Trombert-Paviot, B. Critical analysis of French DRG based information system (PMSI) databases for the epidemiology of cancer: a longitudinal approach becomes possible. Rev Epidemiol Sante Publique. 2011;59(1):53-8.

18. Yetano Laguna, J; López Arbeloa, G; Guajardo Remacha, J; Barriola Lerchundi, MT; Aguirre Larracoechea, U. Calidad de la codificación de los procedimientos quirúrgicos y su influencia en los grupos relacionados con el diagnóstico. Papeles Médicos 2005;15(1) [Consultada el 23.11.2011] Disponible en http://sedom.es/wpcontent/themes/sedom/pdf/4cbd55a040f7ecalidad.pdf

19. Yetano, J; Izarzugaza, I; Aldasoro, E; Ugarte, T; López-Arbeloa, G; Aguirrea, U. Calidad de las variables administrativas del conjunto Mímino Básico de Datos del Oskadietza-Servicio Vasco de Salud. Rev Calidad Asistencial. 2008;23(5):216-21. 
Tabla 1: variables del estudio.

\begin{tabular}{|l}
\hline VARIABLES FINALES \\
\hline Sociodemográficas: \\
- $\quad$ Edad. \\
- $\quad$ Sexo (hombre o mujer).
\end{tabular}

Variables control:

- Diagnóstico principal (CIE-9CM).

- Procedimiento principal (CIE-9CM).

- Motivo de alta (curación, alta domicilio, traslado a otro centro, alta voluntaria fallecimiento).

- Servicio (hospitalización, UCA -Unidad de cirugía sin ingreso-).

- Estancias totales (1 estancia equivale a un ingreso igual o superior a 24 horas. En aquellos casos en los que la estancia sea inferior a 24 horas, se contabilizará como 0,5 estancia).

- Estancia preoperatorio (no de estancias desde el ingreso hasta la intervención).

- Estancia postoperatorio (no de estancias desde la intervención hasta el alta hospitalaria).

- Mes ingreso (nombre del mes).

- Reingreso (según datos facilitados por Unión de Mutuas) (si, no).

- Reintervención (si, no).

- Tipo de anestesia (general, combinada general + regional, regional, local + sedación).

- Tipo de ingreso (urgente con intervención, urgente sin intervención, programado con intervención, programado sin intervención).

- Origen (Unión de Mutuas accidente laboral, Unión de Mutuas contingencia común, Unión de Mutuas enfermedad profesional, Otros, Corporación accidente de trabajo, Corporación enfermedad profesional).

- Tipo de lesión (accidente laboral, enfermedad común, in itínere tráfico, enfermedad profesional). 
Tabla 2. Códigos diagnósticos con mayor número de casos

\begin{tabular}{|c|c|c|c|c|c|c|}
\hline CIE-9CM & Descripción & $\mathbf{n}$ & $\%$ & $\mathrm{EM}^{\mathrm{a}}$ & EM preop ${ }^{b}$ & EM post ${ }^{\mathrm{c}}$ \\
\hline 836.0 & Desgarro de cartílago o menisco interno de la rodilla-actual & 49 & 12.25 & $1(+/-0)$ & 0 & $1(+/-0)$ \\
\hline 717 & Trastorno interno rodilla & 26 & 6.50 & $1(+/-0)$ & 0 & $1(+/-0)$ \\
\hline 719.46 & Dolor articular-pierna & 16 & 4 & $1(+/-0)$ & 0 & $1(+/-0)$ \\
\hline 844 & Esguinces y torceduras de rodilla y pierna & 16 & 4 & $1(+/-0)$ & 0 & $1(+/-0)$ \\
\hline 924.8 & Contusión de múltiples sitios, ncoc & 10 & 2.50 & $2,1(+/-3,47)$ & 0 & $2,1(+/-3,47)$ \\
\hline 726.2 & Otras alteraciones región hombro ncoc & 10 & 2.50 & $1,2(+/-0,42)$ & $0,2(+/-0,42)$ & $1(+/-0)$ \\
\hline 813.41 & Fractura de colles-cerrada & 9 & 2.25 & $2,55(+/-1,66$ & $1(+/-1,41)$ & $1,55(+/-1,33$ \\
\hline 813.4 & Fractura de extremo inferior de radio y cubito-cerrada & 9 & 2.25 & $1,77(+/-1,3)$ & $0,44(+/-1,01$ & $1,33(+/-057)$ \\
\hline 924.11 & Contusion de rodilla & 8 & 2 & $1(+/-0)$ & 0 & $1(+/-0)$ \\
\hline 844.1 & Esguinc/torcedura de ligamento colateral mediano de rodilla & 8 & 2 & $1(+/-0)$ & 0 & $1(+/-0)$ \\
\hline 717.5 & Alteración menisco ncoc & 7 & 1.75 & $1(+/-0)$ & 0 & $1(+/-0)$ \\
\hline 1000 & En estudio & 7 & 1.75 & $1(+/-0)$ & 0 & $1(+/-0)$ \\
\hline 844.2 & Esguince/torcedura de ligamento cruciforme de rodilla & 7 & 1.75 & $2,857(+/-2,67)$ & $0,14(+/-0,37)$ & $2,71(+/-2,56)$ \\
\hline 923.00 & Contusión de la región del hombro & 6 & 1.50 & $1,33(+/-0,51)$ & 0 & $1,33(+/-0,51)$ \\
\hline 719.4 & Dolor articular & 6 & 1.50 & $1(+/-0)$ & 0 & $1(+/-0)$ \\
\hline 550 & Hernia inguinal & 6 & 1.50 & $1(+/-0)$ & 0 & $1(+/-0)$ \\
\hline 727.62 & Ruptura tendon biceps & 6 & 1.50 & $1(+/-0)$ & 0 & $1(+/-0)$ \\
\hline 717.83 & Antigua dehiscencia ligamento cruzado anterior & 4 & 1 & $1,25(+/-0,55)$ & 0 & $1,25(+/-0,55)$ \\
\hline 923.10 & Contusion del antebrazo & 4 & 1 & $24(+/-0)$ & $4,5(+/-3,1)$ & $19,5(+/-3,1)$ \\
\hline 836.1 & Desgarro de cartílago o menisco externo de la rodilla-actual & 4 & 1 & $1(+/-0)$ & 0 & $1(+/-0)$ \\
\hline 719.41 & Dolor articular- hombro & 4 & 1 & $1(+/-0)$ & 0 & $1(+/-0)$ \\
\hline 813.05 & Fractura de cabeza de radio-cerrada & 4 & 1 & $1(+/-0)$ & 0 & $1(+/-0)$ \\
\hline 815.0 & Fractura de hueso(s) metacarpiano(s)-cerrada & 4 & 1 & $1(+/-0)$ & 0 & $1(+/-0)$ \\
\hline 823 & Fractura de tibia y peroné & 4 & 1 & $4(+/-2,94)$ & $1,15(+/-1)$ & $3(+/-1,82)$ \\
\hline
\end{tabular}

a) EM: estancia media; b) EMpreop: estancia media preoperatoria;c) EM post: estancia media postoperatoria.

Tabla 3. Mayor variabilidad en estancia media (EM) total, EM preoperatoria y EM postoperatoria.

\begin{tabular}{|c|c|c|c|c|c|c|c|c|c|}
\hline CIE-9CM & Descripción & EM & Ds & P0\% & $\mathbf{P} 25 \%$ & P50\% & P75\% & P100\% & $\mathbf{n}$ \\
\hline \multicolumn{10}{|c|}{ Mayor variabilidad en la estancia media total } \\
\hline 808 & Fractura de pelvis & 25 & 9.84 & 17 & 19.5 & 22 & 19 & 36 & 3 \\
\hline 823.20 & Fractura de diáfisis de tibia-cerrada & 4 & 4.24 & 1 & 2.5 & 4 & 5.5 & 7 & 2 \\
\hline 823.3 & Fractura de diáfisis de tibia/peroné-abierta & 3.5 & 3.53 & 1 & 2.5 & 3.5 & 4.75 & 8 & 2 \\
\hline 924.8 & Contusión de múltiples sitios, ncoc & 2.1 & 3.47 & 1 & 1 & 1 & 1 & 12 & 10 \\
\hline 823 & Fractura de tibia y peroné & 4 & 2.94 & 1 & 1.75 & 4 & 6 & 7 & 2 \\
\hline \multicolumn{10}{|c|}{ Mayor variabilidad en la estancia media preoperatoria } \\
\hline 808 & Fractura de pelvis & 13.66 & 18.47 & 3 & 3 & 3 & 19 & 35 & 3 \\
\hline 923.10 & Contusión del antebrazo & 4.5 & 3.1 & 1 & 2.5 & 4.5 & 6.5 & 8 & 2 \\
\hline 823.20 & Fractura de diáfisis de tibia-cerrada & 1.5 & 2.12 & 0 & 0.75 & 1.5 & 2.25 & 3 & 4 \\
\hline 813.5 & $\begin{array}{l}\text { Fractura de extremo inferior de radio y } \\
\text { cubito-abierta }\end{array}$ & 0.44 & 1.73 & 0 & 0 & 0 & 1.5 & 3 & 3 \\
\hline 723.1 & Cervicalgia & 1.66 & 1.52 & 0 & 1 & 2 & 2.5 & 3 & 3 \\
\hline \multicolumn{10}{|c|}{ Mayor Variabilidad en la Estancia media postoperatoria } \\
\hline 808 & Fractura de pelvis & 11.33 & 9.29 & 1 & 7.5 & 14 & 16.5 & 19 & 3 \\
\hline 924.8 & Contusión de múltiples sitios, ncoc & 2.1 & 3.47 & 1 & 1 & 1 & 1 & 12 & 10 \\
\hline 923.10 & Contusión del antebrazo & 19.5 & 3.1 & 16 & 15.50 & 19.5 & 21.5 & 23 & 4 \\
\hline 823.3 & Fractura de diáfisis de tibia/peroné-abierta & 3 & 2.82 & 1 & 2 & 3 & 5 & 16.5 & 2 \\
\hline 844.2 & $\begin{array}{l}\text { Esguince/torcedura de ligamento cruciforme } \\
\text { de rodilla }\end{array}$ & 2.71 & 2.56 & 1 & 1 & 2 & 3 & 8 & 7 \\
\hline
\end{tabular}

